Research Article

\title{
Verification of Robustness against Noise and Moving Speed in Self-localization Method for Soccer Robot
}

\author{
Yuehang $\mathrm{Ma}^{1, *}$, Kaori Watanabe ${ }^{1}$, Hitoshi Kono ${ }^{2}$, Hidekazu Suzuki ${ }^{2}$ \\ ${ }^{1}$ Graduate School of Engineering, Tokyo Polytechnic University, 1583 Iiyama, Atsugi, Kanagawa 243-0297, Japan \\ ${ }^{2}$ Faculty of Engineering, Tokyo Polytechnic University, 1583 Iiyama, Atsugi, Kanagawa 243-0297, Japan
}

\author{
ARTICLE INFO \\ Article History \\ Received 13 November 2020 \\ Accepted 22 April 2021 \\ Keywords \\ Robustness against noise \\ robustness against moving speed \\ self-localization \\ RoboCup Middle Size League \\ soccer robot \\ genetic algorithm
}

\begin{abstract}
The main focus of the RoboCup competitions is the game of football (soccer), where the research goals concern cooperative multi-robot and multi-agent systems in dynamic environments. In RoboCup, self-localization techniques are crucial for robots to estimate their own position as well as the position of the goal and other robots before deciding on a strategy. This paper presents a self-localization technique using an omnidirectional camera for an autonomous soccer robot. We propose a selflocalization method that uses the information on the white lines of the soccer field to recognize the robot position by optimizing the fitness function using a genetic algorithm. Through experiments we verify the robustness of the proposed method against noise and moving speed.
\end{abstract}

(C) 2021 The Authors. Published by Atlantis Press B.V. This is an open access article distributed under the CC BY-NC 4.0 license (http://creativecommons.org/licenses/by-nc/4.0/).

\section{INTRODUCTION}

The landmark project RoboCup is a well-known international robotics challenge [1]. This project promotes research on Artificial Intelligence (AI) and robotics with the goal of "creating a team of autonomous humanoid robots that can beat the world-champion soccer team" by 2050 [2]. Various technical challenges must be overcome to achieve this goal. Self-localization-which enables a robot to recognize the real-world environment in real time-is a key function that determines the outcome in any competition because it serves as the starting point for the strategic coordinated movements that follow.

Figure 1 shows the RoboCup Middle Size League (MSL) soccer field, which has the widest field size $(12 \times 18 \mathrm{~m})$. Many selflocalization methods using the shape of the white lines drawn on the field have been proposed, including a method using the Hough transform [3] and a method based on error minimization [4]. However, the Hough transform-based method requires a long processing time because calculations other than estimating the self-localization are generated. Error minimization is a local search method, so the initial position needs to be assumed. As a result of these shortcomings, a method using Monte Carlo localization (MCL) [5,6] with a particle filter has been proposed and implemented by many RoboCup teams. However, improving localization accuracy leads to increased processing time.

Corresponding author.Email: yuehang.ma@gmail.com
Furthermore, MCL is known to be relatively vulnerable to the kidnapping problem [7].

In this paper, we describe a real-time self-localization method that applies a Genetic Algorithm (GA) [8] for the RoboCup MSL. We estimate the self-position using information on the image, environment, and field. A search space is formed by model-based matching [9], which collates a search model based on the shape of the white lines (obtained from omnidirectional images) and known field data. A GA is then used for optimization processing. Our proposed method enables the robot's self-position to be identified in real time.

The competition environment is a dynamic system where multiple robots move at high speed, and image noise is generated due to the mutual occlusion of multiple robots. Moreover, the image changes caused by the movements of the robot also affect self-localization, so the robustness of the self-localization method must be evaluated as well. Thus, we conducted experiments to verify the robustness of our method against noise and moving speed.

\section{SELF-LOCALIZATION USING OMNIDIRECTIONAL CAMERA}

We use a white line on the MSL field for self-localization. Our proposed self-localization method generates the search space based on a model-based matching using white line information. The method recognizes the robot position by optimizing the fitness 

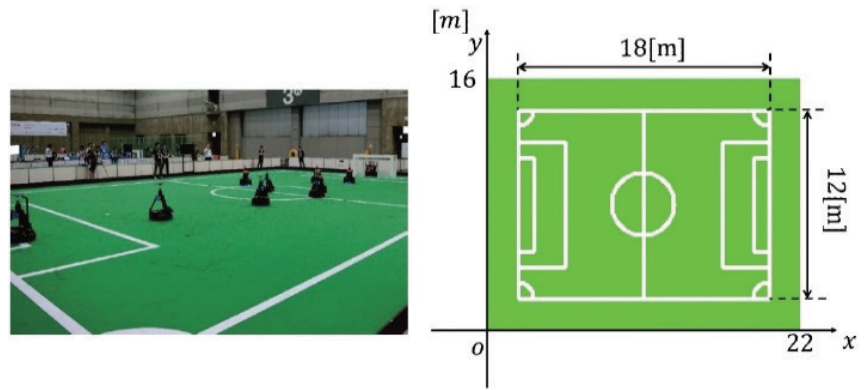

Figure 1 RoboCup MSL soccer field.

function, which is the maximum value at the correct robot position. A GA is utilized to optimize the fitness function.

\subsection{Hardware of Vision System}

The omnidirectional vision system of our robot consists of a camera (FLIR, Flea3), a varifocal lens (Vstone), and a hyperboloidal mirror (Vstone). We developed the vision system, shown in Figure 2, for the RoboCup MSL robot by combining the above elements. The image captured by this vision system is shown in Figure 3a. The image size and frame rate are $512 \times 512$ pixels and 30 frame per millisecond (fps), respectively.

\subsection{Searching Model}

Figure 3 shows the process of creating the search model for the proposed method. First, the detection image of the white line is needed to make the searching model. We obtain the white detection image by applying the converting method of color space from RGB to HSV and to YUV as in Figure $3 b$. Then we generate the field information by orthogonalizing the white line information, shown in Figure 3c. We determine the searching model by thinning down the field information based on the white lines as in Figure $3 \mathrm{~d}$. Finally, we use the thinned model as the searching model for self-localization.

\subsection{Model-based Matching}

The proposed self-localization method generates the search space by model-based matching between geometric information of the white lines on the MSL field and the above-mentioned searching model shown in Figure 4. The evaluation function $F(\tilde{\phi})\{\tilde{\phi}=[\tilde{x}, \tilde{y}, \tilde{\theta}]\}$ of the model-based matching is given as follows:

$$
\boldsymbol{F}(\tilde{\boldsymbol{\phi}})=\sum_{\tilde{\boldsymbol{r}} \in \boldsymbol{S}(\tilde{\phi})} \boldsymbol{p}(\tilde{\boldsymbol{r}})
$$

Here, the movement of the searching model $S$ in the matching area is expressed as $S(\tilde{\phi})$. And, if the pixel value of field image corresponding to the area of the moving model is expressed as $p(\tilde{\boldsymbol{r}})\{\tilde{\boldsymbol{r}}=(\tilde{x}, \tilde{y}), \tilde{\boldsymbol{r}} \in S(\tilde{\boldsymbol{\phi}})\}$. The fitness function $F(\tilde{\boldsymbol{\phi}})$ obtains the maximum value when the position of the searching model corresponds to the correct position of the robot on the MSL field. Then,

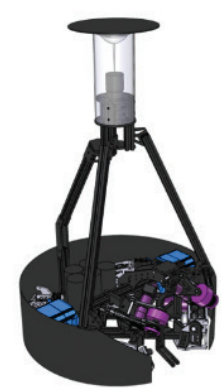

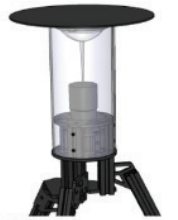

Vision system

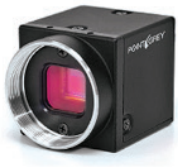

Used Camera
Figure 2 Hardware of vision system. a
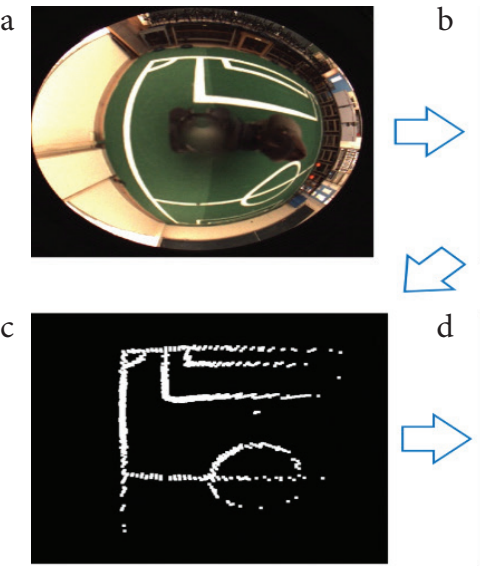

Figure 3 Process of creating search model. (a) Original image from camera. (b) White detection image. (c) Orthogonalized image. (d) Search model.

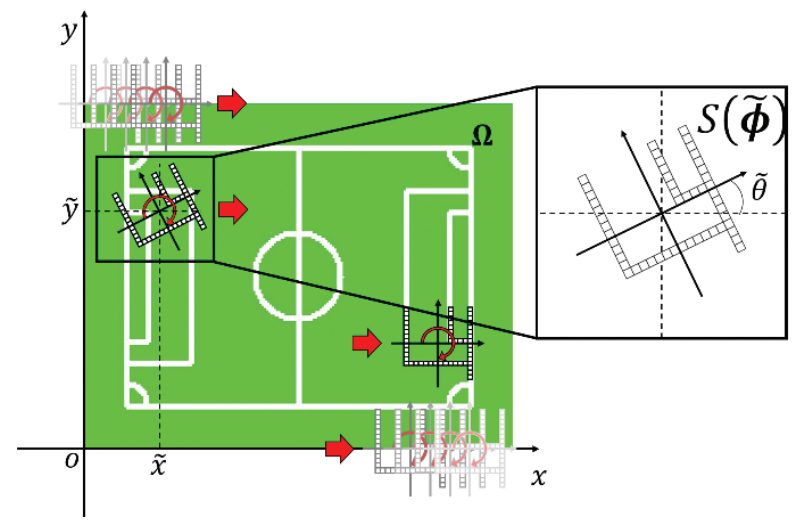

Figure 4 Model-based matching.

the task of detecting robot position is converted to a search task of $\tilde{\phi}$ such that $F(\tilde{\phi})$ is maximized.

\subsection{Genetic Algorithm}

In the proposed self-localization method, we implement a GA to determine the maximum value of the fitness function $F(\tilde{\phi})$. A GA is an example of an artificial intelligence program and is well known as a parallel search and optimization process that mimics natural 
selection and evolution. The proposed method utilizes an elitist model of a GA that preserves the best individual in the population at every generation. The method also utilizes genetic coding using gray code, roulette selection, and one-point crossover. The parameters of the GA process were determined in previous experiments.

\subsection{Verification Experiment}

We performed a self-localization experiment to determine the error between the correct position and detected position on the MSL field to verify the effectiveness of the proposed method. In the verification experiment, the resulting maximum error was $36.1 \mathrm{~cm}$ and the average error was $12.8 \mathrm{~cm}$. These errors are small compared to the field size, indicating that the self-localization could be detected with sufficiently high accuracy.

We conducted an additional verification experiment to confirm the smoothness of the recognized position in an actual environment, in which the robot moved along a test path on the field shown on the left side of Figure 5. The results of the self-localization are shown on the right side of the figure, where the black dots represent the detected position at each camera frame. We also recorded the $x, y$-coordinates of the detected position at each frame number on the test path, shown in Figure 6. The results show that the selflocalization result did not deviate significantly, and the robot's coordinates of its detected position are continuous.

\section{VERIFICATION OF ROBUSTNESS AGAINST NOISE}

The accuracy measured thus far is the result of an ideal environment in which there are no other robots on the soccer field. However, in a real game environment, up to 10 robots may exist on the same soccer field. In addition to the robots, people such as the referee and the line referee are on the field at the same time. The robot may not be able to recognize its own position correctly because the people and the other robots become occlusions. Therefore, we

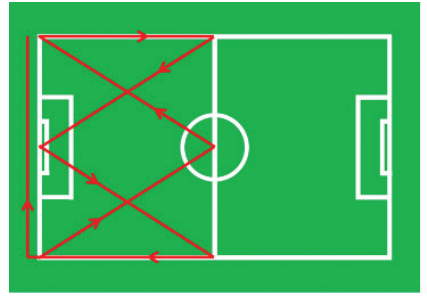

Test path

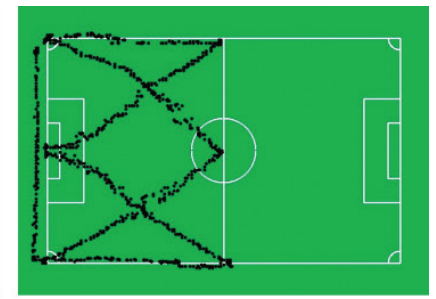

Result of self-localization
Figure 5 Result of self-localization on test path.

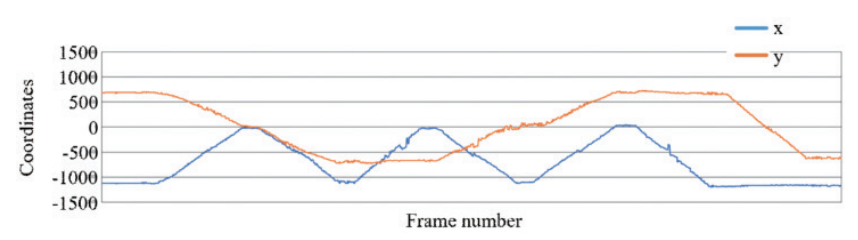

Figure 6 Coordinates of detected position at each frame number. conducted additional experiments to verify the robustness of the proposed method against varying amounts of noise.

\subsection{Experiment}

In a real environment, a variety of noises may appear in the image, so qualitative experiments cannot be performed. Here we perform verification experiments by adding artificial noise.

We conducted the experiments at the seven locations on the MSL field indicated in Figure 7. These seven locations are characteristic points on the field. Taking the center of the panoramic image as the center of the circle, we set the fan-shaped area with a center angle of $30^{\circ}$ as noise, shown in Figure 8. By changing the position and amount of noise, we can simulate different noise conditions and verify the results of self-localization. In the field of research using omnidirectional cameras, the verification method using fan shape noise as described above is often used [10].

Figure 8 shows the changes in the amount of noise at the position $\mathrm{D}$ in Figure 7, and the changes in angle are shown in Figure 9. The image in the left of Figure 8 is the original image without any noise. We verified the error in the self-localization results measured at the angle and number of various noise at each coordinate location on the MSL field. The results of each measurement position are summarized in Table 1. For the position deviation caused by the noise

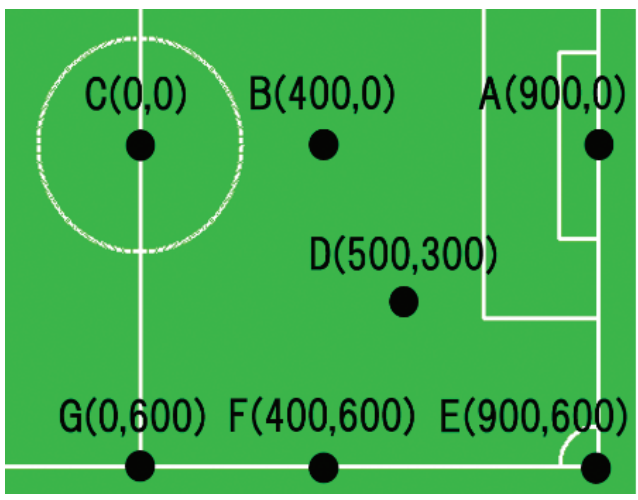

Figure 7 Experiment location.

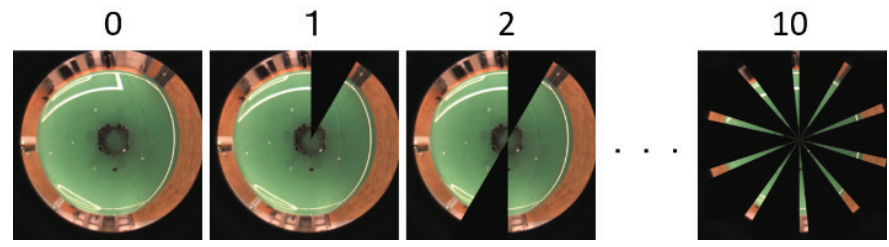

Figure 8 Varying amounts of noise.
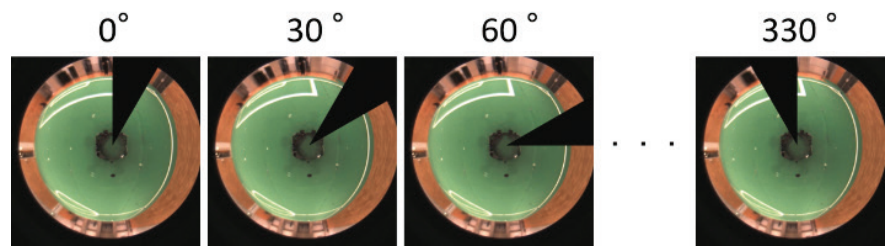

Figure 9 Noise at different angles. 
Table 1 Comparison of detection error

\begin{tabular}{|c|c|c|c|c|c|c|c|c|c|c|c|}
\hline & $\frac{\text { Error }[\mathrm{cm}]}{\text { Rate }}$ & \multicolumn{10}{|c|}{ Noise num } \\
\hline \multirow{10}{*}{ Point } & A & 28.3 & 28.3 & 28.3 & 28.3 & 150.3 & 22.4 & 72.8 & 1510.0 & 28.3 & 131.5 \\
\hline & B & 14.1 & 14.1 & 14.1 & 14.1 & 31.6 & 14.1 & 41.2 & 22.4 & 648.5 & 1164.8 \\
\hline & & $0.00 \%$ & $0.00 \%$ & $0.00 \%$ & $0.00 \%$ & $0.00 \%$ & $0.00 \%$ & $0.00 \%$ & $0.00 \%$ & $50.00 \%$ & $100.00 \%$ \\
\hline & $\mathrm{C}$ & 10.0 & 14.1 & 0.0 & 10.0 & 14.1 & 10.0 & 14.1 & 10.0 & 14.1 & 10.0 \\
\hline & & $0.00 \%$ & $0.00 \%$ & $0.00 \%$ & $0.00 \%$ & $0.00 \%$ & $0.00 \%$ & $8.33 \%$ & $66.67 \%$ & $75.00 \%$ & $100.00 \%$ \\
\hline & E & 22.4 & 22.4 & 22.4 & 58.3 & 1650.5 & 22.4 & 22.4 & 31.6 & 31.6 & 31.6 \\
\hline & & $0.00 \%$ & $0.00 \%$ & $0.00 \%$ & $33.33 \%$ & $8.33 \%$ & $0.00 \%$ & $0.00 \%$ & $0.00 \%$ & $0.00 \%$ & $0.00 \%$ \\
\hline & $\mathrm{F}$ & 30.0 & 30.0 & 41.2 & 14.1 & 20.0 & 40.0 & 20.0 & 40.0 & 41.2 & 370.1 \\
\hline & & $0.00 \%$ & $0.00 \%$ & $0.00 \%$ & $0.00 \%$ & $0.00 \%$ & $0.00 \%$ & $0.00 \%$ & $0.00 \%$ & $0.00 \%$ & $66.67 \%$ \\
\hline & G & 10.0 & 10.0 & 40.0 & 120.0 & 40.0 & 10.0 & 820.1 & 900.1 & 890.1 & 900.1 \\
\hline
\end{tabular}

at different angles, we took the average value combined with the deviation value caused by the amount of noise. The bold text in the table indicates that the error value of the self-localization exceeds the width of a robot $(50 \mathrm{~cm})$, meaning that the robot has lost its exact position.

\subsection{Experiment Results}

As shown in the results in Table 1, when the amount of noise increases, the self-localization error also increases. Generally, when the number of noises is greater than 7 , it will cause large errors except for special locations, i.e., where there are more than two features in the image obtained by the robot (Locations $\mathrm{B}$ and F) and when the robot is in the center circle (Location $\mathrm{C}$ ), shown in Figures 10 and 11, respectively. Locations $\mathrm{E}$ and $\mathrm{G}$ also have errors when the noise is low. This may be because the robot was on the vertical white line, and the noise obscured all of the white line information, shown in Figure 12. Overall, the results show that when the number of noise is less than 3 , the selflocalization error is very small, and when the noise increases to 4 , the maximum self-localization error is $120 \mathrm{~cm}$. In a real game environment, the probability of more than four noises existing at the same time is very low.

\section{VERIFICATION OF ROBUSTNESS AGAINST MOVING SPEED}

Genetic algorithm searching obtains the optimum value of fitness function by changing and converging the generation continuously against a fixed search space such as a static image. However, when processing a dynamic image, the target position changes at every moment. The target position $\phi p$ in the image changes and is represented by $\phi p(t)$ as the time function. Realtime processing, which calculates within $33 \mathrm{~ms}$ per frame is very important for recognizing target position $\phi p(t)$ without delay. In this research, the proposed method outputs the target position obtained by the maximum value of the genetic fitness function at every frame change.
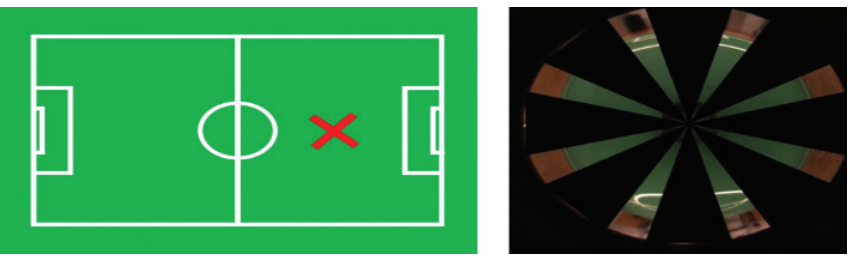

Figure 10 Eight noise patterns at position B.
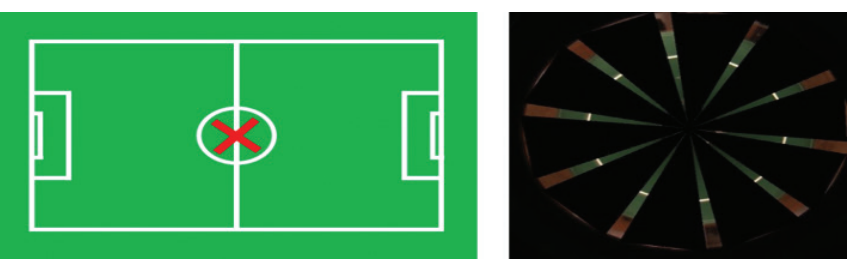

Figure 1110 noise patterns at position C.
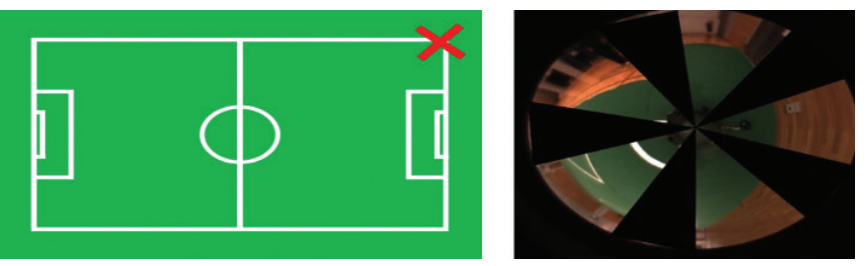

Figure 12 Five noise patterns at position E.

\subsection{Inheritance of Genetic Information}

An example of the output fitness value from the general GA search is shown in Figure 13, where the vertical line represents the timing of frame-to-frame switching. In this figure, the fitness value drops significantly because the best individual is reset with every frame change and the search restarts from a very low initial fitness value. The proposed method uses the genetic information of the previous frame as the initial genetic information of the next frame at every frame change. The resulting output of the proposed method is shown in Figure 14. As seen in the figure, a high fitness value is maintained by inheriting the individual information. 


\subsection{Experiment Results}

To verify the method's robustness to moving speed, we conducted experiments in which we measured the robot's fitness value and self-localization accuracy at different robot speeds. The robot moves forward on test paths A-C, shown in Figure 15, at a speed of $1-3 \mathrm{~m} / \mathrm{s}$.

The result of test path $\mathrm{A}$ is shown in Figures 16 and 17. In Figure 16, the vertical axis represents the fitness value and the horizontal axis represents the distance from the starting point. To verify the relationship between accuracy and moving speed, we picked three areas surrounded by rectangles in Figure 16, which are rearranged in Figure 17. In Figure 17, I-III are the respective positions and frames at each speed.

Here, the position error is represented with a bar graph. As shown in Figure 17, the self-localization error does not change greatly as moving speed changes. The maximum error is about $13 \mathrm{~cm}$, which

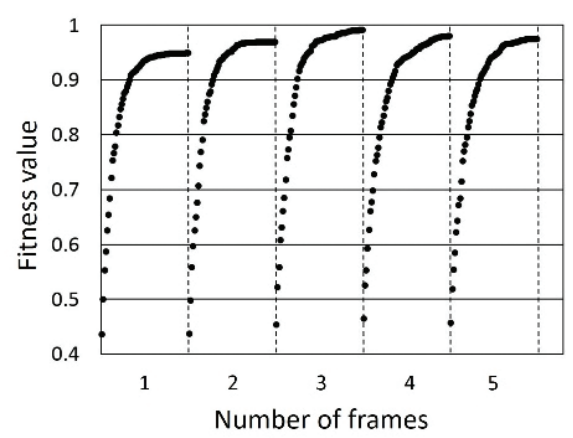

Figure 13 Output of fitness value from general GA search.

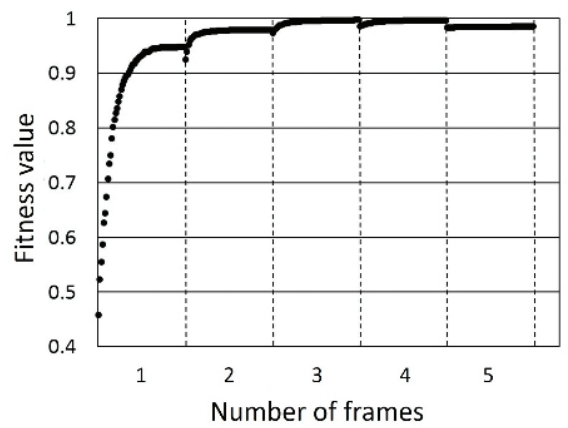

Figure 14 Output of fitness value using proposed method.

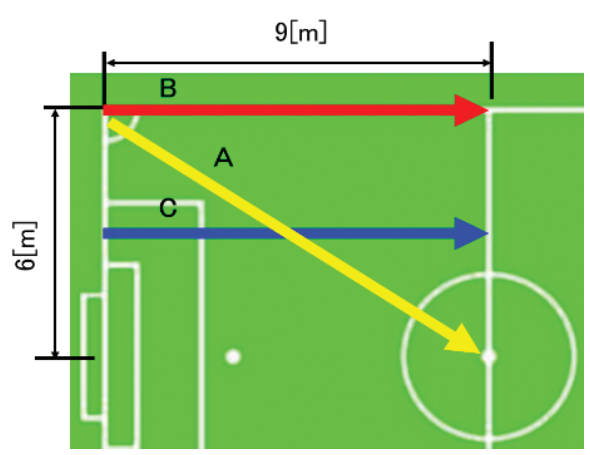

Figure 15 Test paths A-C.

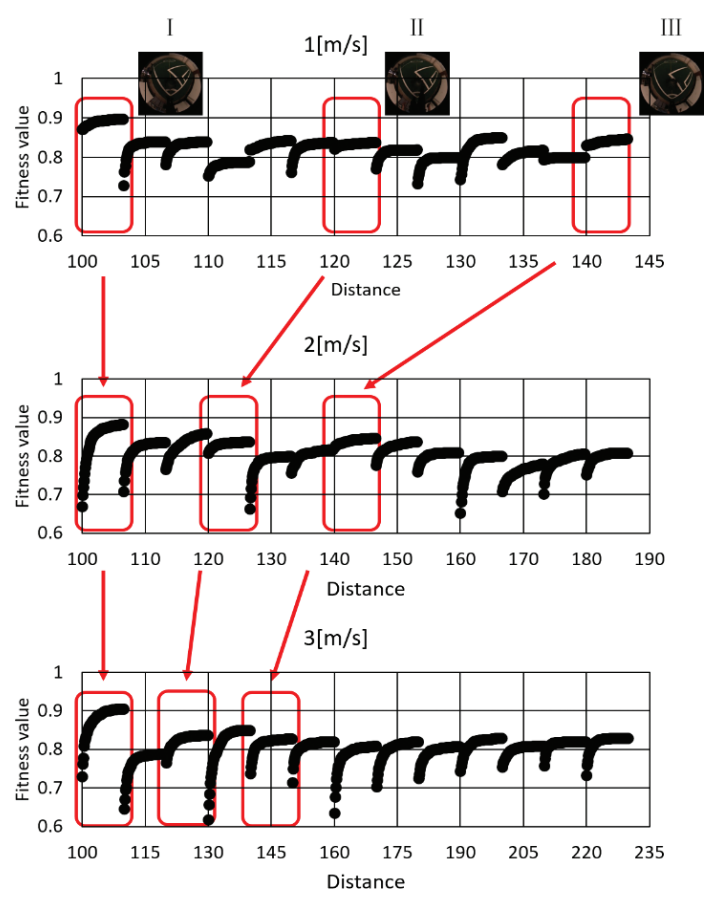

Figure 16 Results of fitness value at each moving speed (test path A).
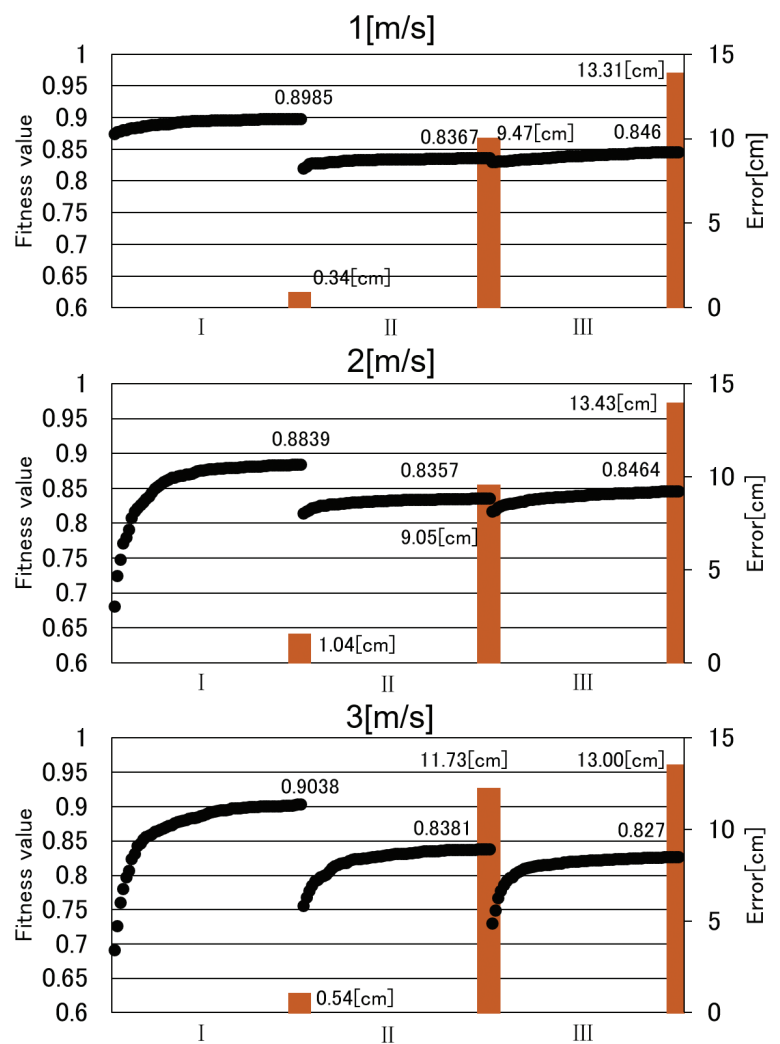

Figure 17 Self-localization error at each moving speed (test path A).

is small enough compared to the size of the MSL field. Test paths $\mathrm{B}$ and $\mathrm{C}$ yielded similar results to test path $\mathrm{A}$; the maximum errors were 9.9 and $10.12 \mathrm{~cm}$ for $B$ and $C$, respectively. This shows that the proposed method is robust against changes in the robot's moving speed within $1-3 \mathrm{~m} / \mathrm{s}$. 


\section{CONCLUSION}

We proposed a self-localization method that generates the search space based on a model-based matching with the white line information of the RoboCup MSL soccer field. The method recognizes the robot position by optimizing the fitness function using a genetic algorithm. We verified the effectiveness and accuracy of the proposed self-localization method using the GA in an actual environment. Furthermore, we experimentally evaluated the robustness of the proposed method against noise and moving speed and confirmed that it is sufficient for RoboCup soccer games.

\section{CONFLICTS OF INTEREST}

The authors declare they have no conflicts of interest.

\section{REFERENCES}

[1] RoboCup Federation official website. Available from: https:// www.robocup.org/.

[2] M. Asada, H. Kitano, I. Noda, M. Veloso, RoboCup: Today and tomorrow-What we have learned, Artificial Intelligence 110 (1999), 193-214.

[3] T. Peijnenburg, J.v. Eijck, et al., VDL Robot Sports Team Description Paper, 2016. Available from: https://www. robotsports.nl/index.php/en/documents/qualificationmaterial-2016.

[4] M. Lauer, S. Lange, M. Riedmiller, Calculating the perfect match: an efficient and accurate approach for robot self-localization, in: A. Bredenfeld, A. Jacoff, I. Noda, Y. Takahashi (Eds.), RoboCup 2005: Robot Soccer World Cup IX, RoboCup 2005. Lecture Notes in Computer Science, vol. 4020, Springer, Berlin, Heidelberg, 2006, pp. 142-153.

[5] A.A.F. Nassiraei, Y. Kitazumi, S. Ishida, H. Toriyama, H. Ono, K. Takenaka, et al., Hibikino-Musashi Team Description Paper, 2011.

[6] Y. Komoriya, R. Hashimoto, K. Miyoshi, Y. Ogawa, D. Sakakibara, J. Sawada, et al., WinKIT 2009 Team Description Paper, 2009.

[7] R. Ueda, T. Arai, K. Sakamoto, T. Kikuchi, S. Kamiya, Expansion resetting for recovery from fatal error in Monte Carlo localization - comparison with sensor resetting methods, 2004 IEEE/RSJ International Conference on Intelligent Robots and Systems (IROS), IEEE, Sendai, Japan, 2004, pp. 2481-2486.

[8] D.E. Goldberg, Genetic algorithms in search, optimization and machine learning, Addison-Wesley Longman Publishing Co., Inc., MA, USA, 1989, p. 372.

[9] K. Watanabe, Y. Ma, H. Suzuki, Real-time self-localization using model-based matching for autonomous robot of RoboCup MSL, J. Robot. Netw. Artif. Life 7 (2020), 98-102.

[10] H. Lu, X. Li, H. Zhang, Z. Zheng, Robust place recognition based on omnidirectional vision and real-time local visual features for mobile robots, Adv. Robot. 27 (2013), 1439-1453.

\section{AUTHORS INTRODUCTION}

\section{Mr. Yuehang Ma}

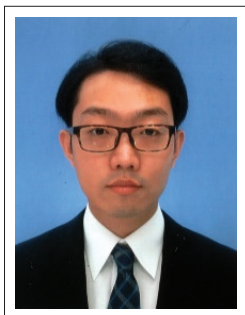

He received his B.S. and M.S. degree in Engineering in 2018 and 2020 from the Faculty of Engineering, Tokyo Polytechnic University in Japan. He is acquiring the PhD in Tokyo Polytechnic University.

\section{Dr. Hitoshi Kono}

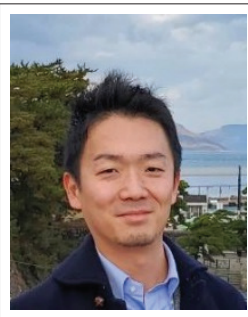

He received his B.S., M.S. and $\mathrm{PhD}$ degrees from Tokyo Denki University, Japan, in 2008, 2010 and 2015 respectively. He joined Fujitsu Limited from 2010 to 2011. In 2015, he joined the Department of Precision Engineering, The University of Tokyo as a Project Researcher. Since 2017, he has been an Assistant Professor in the Department of Electronics and Mechatronics, Tokyo Polytechnic University. His research interests include disaster response robotics, transfer reinforcement learning and it's applications. He is a member of RSJ, JSAI, and IEEE R\&A.

\section{Ms. Kaori Watanabe}

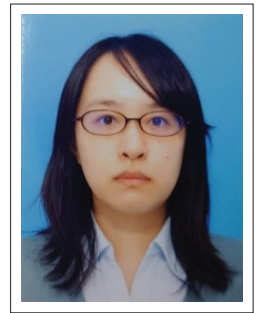

She received her Master's degree from the Department of System Electronics and Information Science, Tokyo Polytechnic University, Japan in 2013. She is currently a Doctoral course student in Tokyo Polytechnic University, Japan.

\section{Dr. Hidekazu Suzuki}

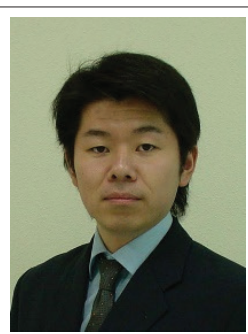

He is an Associate Professor of Faculty of Engineering at Tokyo Polytechnic University in Japan. $\mathrm{He}$ graduated from the Department of Mechanical Engineering, Fukui University, in 2000. He received his D. Eng. degree in System Design Engineering from Fukui University in 2005. His research interest is Robotics. 